\title{
Plasmon measurements with a seeded x-ray laser
}

\author{
L. B. Fletcher, ${ }^{a}$ E. Galtier, ${ }^{a}$ P. Heimann, ${ }^{a}$ H. J. Lee, ${ }^{a}$ B. Nagler, ${ }^{a}$ J. Welch, ${ }^{a}$ U. \\ Zastrau, ${ }^{\mathrm{a}, \mathrm{b}}$, J. B. Hastings, ${ }^{\mathrm{a}}$ and S. H. Glenzer ${ }^{\mathrm{a},}$ \\ ${ }^{a}$ SLAC National Accelerator Laboratory, \\ 2575 Sand Hill Road, MS 72, Menlo Park, CA 94025, USA \\ ${ }^{b}$ Helmholtz-Institut Jena, \\ Fröbelstieg 3, 07743 Jena, Germany \\ E-mail: glenzereslac.stanford.edu
}

\begin{abstract}
Plasmon measurements hold great promise for providing highly accurate data on the physical properties of plasmas in the high-energy density physics regime. To this end we demonstrate in recent experiments at the Linac Coherent Light Source the first spectrallyresolved measurements of plasmons using a seeded $8-\mathrm{keV}$ x-ray laser beam. Forward $\mathrm{x}$-ray Thomson scattering spectra from isochorically heated solid aluminum show a well-resolved plasmon feature that is down-shifted in energy by $19 \mathrm{eV}$ from the incident $8 \mathrm{keV}$ elastic scattering feature. In this spectral range, the simultaneously measured backscatter spectrum shows no spectral features indicating observation of collective plasmon oscillations on a scattering length comparable to the screening length. This technique is a prerequisite for Thomson scattering measurements in compressed matter where the plasmon shift is a sensitive function of the free electron density and where the plasmon intensity provides information on temperature.
\end{abstract}

KEYWORDS: Thomson scattering, Spectrometers, Plasma diagnostics - interferometry, spectroscopy and imaging.

\footnotetext{
${ }^{*}$ Corresponding author.
} 


\section{Contents}

1. Introduction $\quad 1$

2. X-ray Thomson scattering in solid-density plasmas $\quad 2$

$\begin{array}{ll}2.1 \text { Photon requirements } & 2\end{array}$

$\begin{array}{ll}2.2 \text { Resolution requirements } & 3\end{array}$

3. Experiment 4

3.1 Seeded LCLS x-ray beam 4

3.2 Experimental geometry 5

3.3 Highly Annealed Pyrolytic Graphite (HAPG) spectrometer $\quad 6$

$\begin{array}{ll}\text { 4. Results and Discussions } & 7\end{array}$

$\begin{array}{lr}\text { 5. Conclusions } & 9\end{array}$

\section{Introduction}

With the advent of the Linac Coherent Light Source (LCLS) x-ray laser [1] and the commissioning of the Matter of Extreme Conditions (MEC) end station [2] an unprecedented experimental capability has become available to explore extreme matter conditions with accurate pump-probe measurements [3]. Experiments that apply spectrally resolved x-ray scattering to probe plasma conditions of compressed solids offer novel techniques to determine the physical properties of matter at mega bar pressures. The ultrafast temporal resolution provided by the LCLS x-ray source and the high repetition rate of these experiments make studies of high-pressure phase transitions [4,5], observations of novel structural properties [6], or direct measurements of material strain rates highly attractive.

For this purpose, the MEC instrument is equipped with one ultra-short pulse laser and two nanosecond laser beams that drive the material into extreme matter conditions, for example, by isochoric heating or by launching shock waves that propagate through solid foil targets. In these conditions, spectrally resolved x-ray Thomson scattering measurements in the noncollective (backward) scattering regime provide information of the microscopic physics by measuring the free electron distribution function. On the other hand, in the collective (forward) scattering regime, plasmon (Langmuir) [7-9] or ion acoustic oscillations are observed [10]. In particular, the plasmon scattering spectrum is of fundamental interest because it holds promise to determine plasma parameters and the physical properties from first principles [11]. This is particularly relevant for solid densities and above where the plasma is often closely coupled and standard theoretical approximations that have been developed for solids or ideal plasmas are not applicable. On the other hand, knowledge of dense plasma conditions are important for warm dense matter studies and potential future applications where contemporary physics pursues 
questions related to particle acceleration [12-13], inertial confinement fusion [14-15], and laboratory astrophysics [16-17].

LCLS provides a powerful penetrating $x$-ray beam with unique properties to probe the conditions in these short-lived hot dense states of matter. The beam delivers approximately $10^{12}$ $\mathrm{x}$-ray photons in a micron-scale focal spot allowing measurements with high spectral resolution of $\Delta \mathrm{E} / \mathrm{E}=10^{-4}$ (in a seeded beam mode), high wave-number resolution of $\Delta \mathrm{k} / \mathrm{k}=10^{-2}$, and high temporal resolution of 20-50 fs. Consequently, by employing highly efficient curved crystal spectrometers, the plasmon spectrum can be observed in single shots.

In this study, experiments have been performed in single shot mode and at high repetition rate of $115 \mathrm{~Hz}$. The latter allowed us to measure quasi noise-free plasmon spectra within 7 seconds that determine the plasmon dispersion and intensity for warm solid-density aluminum. The plasmon shift of $19 \mathrm{eV}$ yields $\mathrm{n}_{\mathrm{e}}=1.8 \times 10^{23} \mathrm{~cm}^{-3}$ expected for $\mathrm{Z}=3$ and temperatures less than $1 \mathrm{eV}$. These findings demonstrate the plasmon scattering capability at LCLS and will motivate future investigations in hot compressed matter.

\section{X-ray Thomson scattering in solid-density plasmas}

\subsection{Photon requirements}

Experiments that require observations of the complete plasmon spectrum in a single shot require powerful light sources. This requirement can be derived from the Thomson scattering cross section of $\sigma=8 \pi / 3 r_{0}^{2}=0.665 \times 10^{-24} \mathrm{~cm}^{2}$, with $r_{0}=2.8 \times 10^{-15} \mathrm{~m}$ being the classical electron radius. Past experiments in the high-density regime employed laser-produced $\mathrm{x}$-ray sources in close proximity to the dense plasma $[3,7-9,11]$. They used laser energies of $E_{L}=5 \mathrm{~kJ}$ that is converted to $\mathrm{x}$-ray line radiation with an efficiency of order $1 \%$ producing $50 \mathrm{~J}$ of $8 \mathrm{keV}$ $\mathrm{x}$-rays in a single He- $\alpha$ emission line. However, only $1 \%$ of the He- $\alpha$ radiation would reach the high-density plasma and only $20 \%$ of these x-rays contributed to a scattering spectrum that was temporally gated to $0.2 \mathrm{~ns}$. Thus, the total $\mathrm{x}$-ray energy at the sample that contributed to a scattering spectrum can be estimated through $E_{X-\text { ray }}=E_{L} \eta_{\text {X-ray }} d \Omega \delta \tau=5 \times 10^{3} \mathrm{~J} \times 0.01 \times 0.01 \times$ $0.2=0.1 \mathrm{~J}$ or $10^{14} \mathrm{x}$-ray photons. Measurements have been performed on large-scale laser facilities with order of 10 shots per experiment. Although the total number of $\mathrm{x}$-ray photons is significantly larger on large laser facilities, the use of nanosecond laser beams requires the use of gated and heavily filtered microchannelplate detectors with low detection efficiency.

For a nanosecond laser source, applying $10^{14} \mathrm{x}$-ray photons to a dense plasma with electron density of $\mathrm{n}_{\mathrm{e}}=1.8 \times 10^{23} \mathrm{~cm}^{-3}$ and scale length of $100 \mu \mathrm{m}$ results in a scattering fraction of $n_{e} \sigma l /\left(1+\alpha^{2}\right)=0.01$ and $10^{12}$ scattered $\mathrm{x}$-ray photons. Here $\alpha$ is the scattering parameter and is of order 1 for this study, see Eq. (2) below. A crystal spectrometer with a crystal reflectivity of 1 mrad with 1 rad coverage, and a gated microchannelplate detector with $1 \%$ detection efficiency results in a collection fraction of $\eta_{\text {coll }}=0.1 \mathrm{rad} \times 1 \mathrm{mrad} \times 1 \% / 4 \pi=10^{-7}$. Thus the scattering spectrum consists of $\sim 10^{5}$ photons.

Applying the LCLS x-ray laser beam compares favorably to these experiments because the ultrafast laser pulse is focused into the dense plasma. With about $\mathrm{mJ}$ of $\mathrm{x}$-ray energy and $10^{12}$ $\mathrm{x}$-ray photons, using again a scattering fraction of $n_{e} \sigma l /\left(1+\alpha^{2}\right)=0.01$ yields $10^{10}$ scattered $\mathrm{x}$-ray 
photons. There is no requirement for gating and detectors with over $50 \%$ efficiency can be used resulting in a comparable photon estimate of $10^{4}-10^{5}$ photons. Distributed over say 10 spectral bins, the expected signal-to-noise (SNR) ratio due to photon statistics will be 50-100, which is excellent. At LCLS, these measurements can be performed with the additional advantages of greatly improved temporal, spectral and wavenumber resolution.

\subsection{Resolution requirements}

The $\mathrm{x}$-ray scattering process is markedly different from optical scattering as the energy of the incident $\mathrm{x}$-ray photon with frequency $\omega_{0}$ is large enough to give a significant Compton or plasmon shift to the frequency of the scattered radiation. During the scattering process, the incident photons transfer the momentum $h \boldsymbol{k} / 2 \pi$ and the energy $h \omega_{C} / 2 \pi=(h \boldsymbol{k} / 2 \pi)^{2} / 2 m_{e}$ to the electrons. The magnitude of the $\mathbf{k}$-vector is given by

$$
k=|\mathrm{k}|=\frac{4 \pi h c}{E_{0}} \sin \left(\frac{\theta}{2}\right)
$$

with $E_{0}=h \omega_{0} / 2 \pi$ being the energy of the probe $\mathrm{x}$ rays and $\theta$ the scattering angle. Momentum and energy is transferred to free electrons and to electrons whose binding energy is less than $h \omega_{C} / 2 \pi$. With the scattering vector defined by x-ray energy and scattering geometry, the Thomson scattering regime is characterized by the scattering parameter $\alpha$ that is proportional to the ratio of the x-ray probe scale-length to the plasma screening length, $\lambda_{s}$ :

$$
\alpha=\frac{1}{k \lambda_{S}}
$$

For $\alpha<1$, the non-collective regime, spectrally-resolved incoherent Thomson scattering measures the momentum distribution function for free electrons, and hence the electronic temperature. In warm dense matter, however, this spectrum blends with the bound-free scattering spectrum [18-19] that measures the momentum distribution of the bound electrons. Moreover, bound electrons with ionization energies larger than $h \omega_{d} / 2 \pi$ (states deep in the Fermi sphere) cannot be excited, and no energy can be transferred during the scattering process giving rise to an elastic scattering feature. For $\alpha>1$, the collective scattering regime, the scattering is sensitive to temporal correlations between electron motion separated by a screening length, and therefore observes ion-acoustic and electron plasma wave resonances.

The total scattering spectrum results in elastic and inelastic spectral features that are calculated with the dynamic form factor. The free electron contribution is described by $S_{e e}(k, \omega)$, while the un-shifted elastic scattering component at $E_{0}$ that is commonly referred to as Rayleigh peak is described by the first term of the dynamic structure factor [20-22]

$$
S(k, \omega)=\left|f_{I}(k)+q(k)\right|^{2} S_{i i}(\mathrm{k})+Z_{f} S_{e e}(k, \omega)+Z_{c} \int S_{C E}\left(k, \omega-\omega^{\prime}\right) S_{S}\left(k, \omega^{\prime}\right) d \omega^{\prime}
$$

with $Z_{f}$ and $Z_{C}$ the number of free and bound electrons, respectively. The last term of Eq. (3) calculates the bound-free scattering contribution. This expression for the dynamic structure factor allows a few simple approximations. Of interest to this study, $\alpha>1$ results in collective scattering and the plasmon frequency shift from $E_{0}$ is determined by the plasmon dispersion relation and the width is determined by Landau damping and collisional damping processes. 
The shift can be approximated for small values of $k$ using an inversion of Fermi integrals given by Zimmerman [23] that results in a modified Bohm-Gross dispersion relation $[23,24]$

$$
\omega_{p l}^{2}=\omega_{p}^{2}+3 k^{2} v_{t h}^{2}\left(1+0.088 n_{e} \Lambda_{e}^{3}\right)+\left(\frac{\hbar k^{2}}{2 m_{e}}\right)^{2}
$$

where $\omega_{p}=\sqrt{n_{e} e^{2} / \varepsilon_{0} m_{e}}$ is the plasma frequency, $v_{t h}=\sqrt{k_{B} T_{e} / m_{e}}$ is the thermal velocity, and $\Lambda_{e}=h / \sqrt{2 \pi m_{e} k_{B} T_{e}}$ is the thermal wavelength. For solid-density plasma conditions encountered in warm dense matter, Eq. (4) results in energy shifts in the range of $15-50 \mathrm{eV}$ consequently requiring a high-energy x-ray source of $E>3 \mathrm{keV}$ and $\Delta E / E=10^{-3}-10^{-4}$ to both penetrate through the dense plasma and to resolve the plasmon frequency shift. Furthermore, it is noteworthy to realize that simultaneous energy resolution of $\Delta E / E<10^{-4}$ and wavenumber resolution of $\Delta k / k<10^{-1}$ will be needed to resolve plasmon broadening determined by damping processes in the plasma.

\section{Experiment}

\subsection{Seeded LCLS x-ray beam}

In this study, we employed a seeded LCLS x-ray laser beam to achieve a narrow bandwidth and high-energy resolution sufficient to observe plasmon resonances. We further show that the resolution of a SASE beam is not sufficient to meet this objective. Figure 1 shows a schematic of the seeding setup for LCLS [25]. In these experiments, the Linac accelerates electrons with a single bunch charge of $150 \mathrm{pC}$ charge with peak current of $2.91 \mathrm{kA}$ to approximately $13.6 \mathrm{GeV}$ electron beam energy. Within the first 15 undulators the Linac produces Self Amplified Spontaneous Emission (SASE) with about $1 \mathrm{GW}$ power at a wavelength of $1.5 \AA$, at $8 \mathrm{keV}$ x-ray energy. The electron beam is then passed through a 4-m long chicane while the x-ray beam passes through a diamond crystal that Bragg reflects a narrow energy range of $\mathrm{x}$ rays with a bandwidth of $\Delta E / E=0.5-1 \times 10^{-4}$ in the center of the broad SASE spectrum. The beating within the transmitted x-ray spectrum creates a $5 \mathrm{MW}$ trailing monochromatic seed pulse about 20 fs or $6 \mu \mathrm{m}$ behind the broad bandwidth SASE pulse. The electron bunch is delayed slightly, superimposed with the seed pulse and amplified to $10-15 \mathrm{GW}$ in the subsequent 17 undulators.

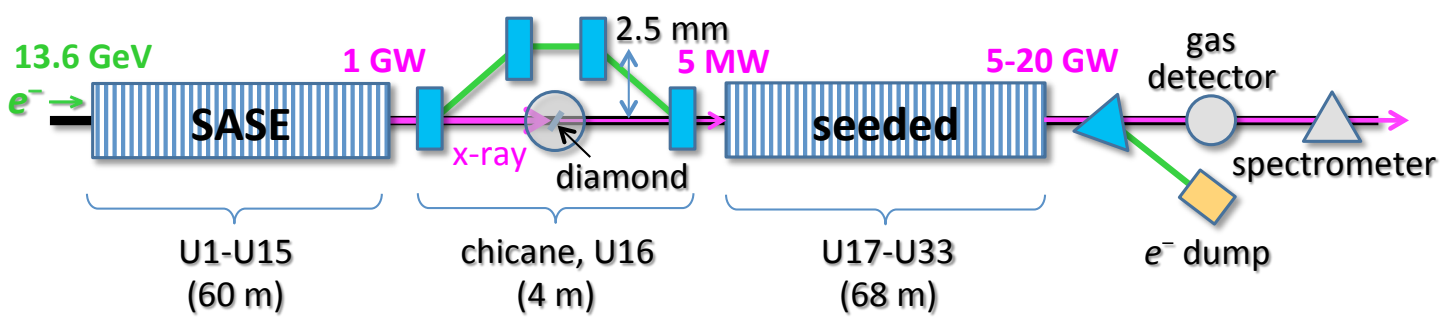

Figure 1. A schematic of the LCLS seeded beam generation is shown producing approximately $0.5 \times 10^{12}$ photons at $8 \mathrm{keV}$ with $\Delta E / E=0.5-1 \times 10^{-4}$ and pulse durations of $30-50 \mathrm{fs}$.

After the undulators and the electron beam dump a 200-m long x-ray transport section will deliver the x-ray beam to the MEC instrument. The transport beam path includes a grazing 
incidence mirror at $2.75 \mathrm{mrad}$ that reflects the beam towards MEC, through collimators, profile monitors, and beam attenuators. This setup provides either a single x-ray pulse or a series of pulses to the instrument. Inside the MEC hutch the beam size is about $1 \times 1 \mathrm{~mm}^{2}$ before Be lenses focus the beam onto the target. In this study, beam foci of $5 \mu \mathrm{m}$ and $10 \mu \mathrm{m}$ have been employed.

\subsection{Experimental geometry}

Figure 2 shows a schematic of the x-ray scattering experiment. The target consists of a $25-$ $\mu \mathrm{m}$ thick aluminum foil mounted in a target holder with $3 \mathrm{~mm}$ diameter access holes. The figure shows an example image of a target that was exposed to $115 \mathrm{~Hz}$ x-ray pulses where the target was moving from left to right during a $1 \mathrm{~s}$ exposure cycle so that each $\mathrm{x}$-ray pulse interacts with a fresh target surface. This target was employed for about 25 measurements. The $13^{\circ}$ forward scattering spectrum from these measurements shows a well-resolved plasmon peak while the $135^{\circ}$ backscattering measurements show no scattering feature in the energy range of the plasmon. Also shown is the seeded LCLS x-ray spectrum measured in separate shots behind the target chamber and without a target. In these experiments, scattering spectra summing over 100 to $700 \mathrm{x}$-ray pulses have been obtained within several minutes. Single shot experiments on laser driven aluminum use a $2 \mu \mathrm{m}$ thick $\mathrm{CH}$ coating that serves as an ablator. In that case, the cool-down time for the optical nanosecond laser determines the repetition rate of 1 shot in about 3 to 7 minutes.

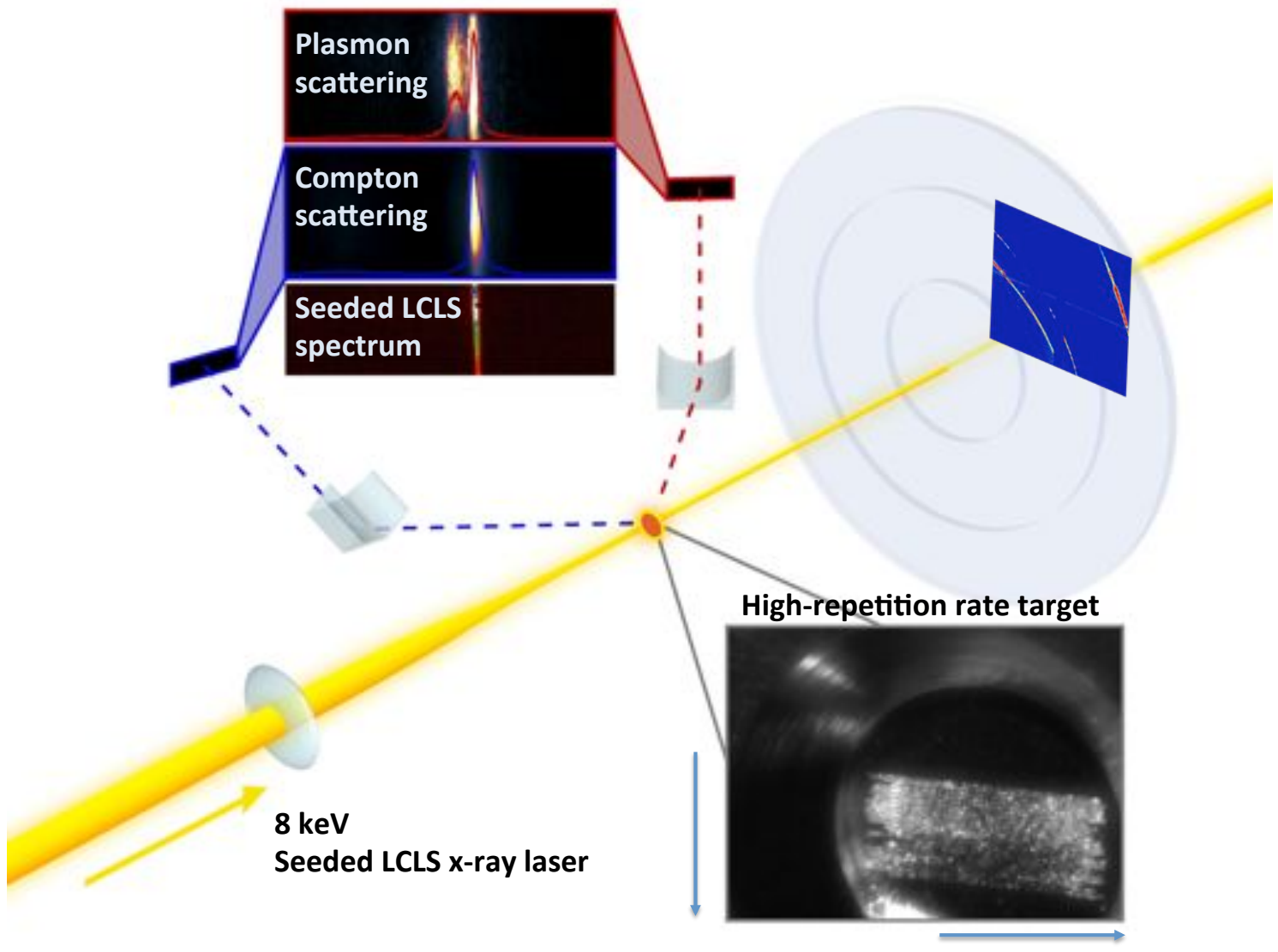

Figure 2. A schematic of LCLS x-ray scattering experiment on solid aluminum is shown together with a target image, the incident LCLS spectrum, x-ray scattering spectra in forward and backward geometry, and forward x-ray diffraction data with Debye-Scherrer rings. 


\subsection{Highly Annealed Pyrolytic Graphite (HAPG) spectrometer}

To maximize the collection fraction, $\mathrm{x}$-ray scattering experiments require spectrometers with extraordinary efficiency of $>0.01$, but with high resolving power of $\Delta \mathrm{E} / \mathrm{E}<10^{-3}$. In the multi-keV $\mathrm{x}$-ray regime, artificial crystals from low-Z materials such as carbon with a mosaic spread of $\sim 0.1^{\circ}$ approach these requirements. To further improve collection efficiency the distances from the crystal to both source and detector are typically chosen to be the same, resulting in mosaic focusing and high spectral resolution $[3,26]$. In addition, a cylindrically bent crystal in von-Hamos geometry will further optimize collection.

In this study, we employ highly-annealed pyrolytic graphite (HAPG) [27-28] that has been shown to provide advantages over previously used HOPG [26,29]. The point-spread function of these spectrometers is dominated by so-called depth broadening. Due to negligible photoabsorption (the attenuation length of $8 \mathrm{keV}$-rays is $C$ is about $1 \mathrm{~mm}$ ), $x$-rays penetrate deep into the crystal until they encounter a crystallite that is properly oriented to reflect the ray. In von-Hamos geometry, the spot in the detector will therefore be broadened in the dispersion plane by $2 \mathrm{D} / \tan \left(\theta_{\mathrm{B}}\right)$ with the crystal thickness $\mathrm{D}$ and the Bragg angle $\theta_{\mathrm{B}}$. Ray tracing simulations for a point source and monochromatic $8 \mathrm{keV} \mathrm{x}$-rays have been performed for HAPG crystals of $30 \times 30 \mathrm{~mm}^{2}$ size and $51.7 \mathrm{~mm}$ radius of curvature $\left(\theta_{\mathrm{B}}=13^{\circ}\right)$. The calculations show that the point spread function of the $40 \mu \mathrm{m}$ thick crystal is about $6 \mathrm{eV}$ wide with $\mathrm{E} / \Delta \mathrm{E}=1300$ suitable for resolving the plasmon shift in dense plasmas. A $100-\mu \mathrm{m}$ thick crystal has lower spectral resolution of $14 \mathrm{eV}$ with $\mathrm{E} / \Delta \mathrm{E}=550$, but results in a higher reflectivity, as determined by the integral over the reflection curve. Some decrease of reflectivity in the high-photon energy tail is observed due to photo-absorption in the crystal volume.

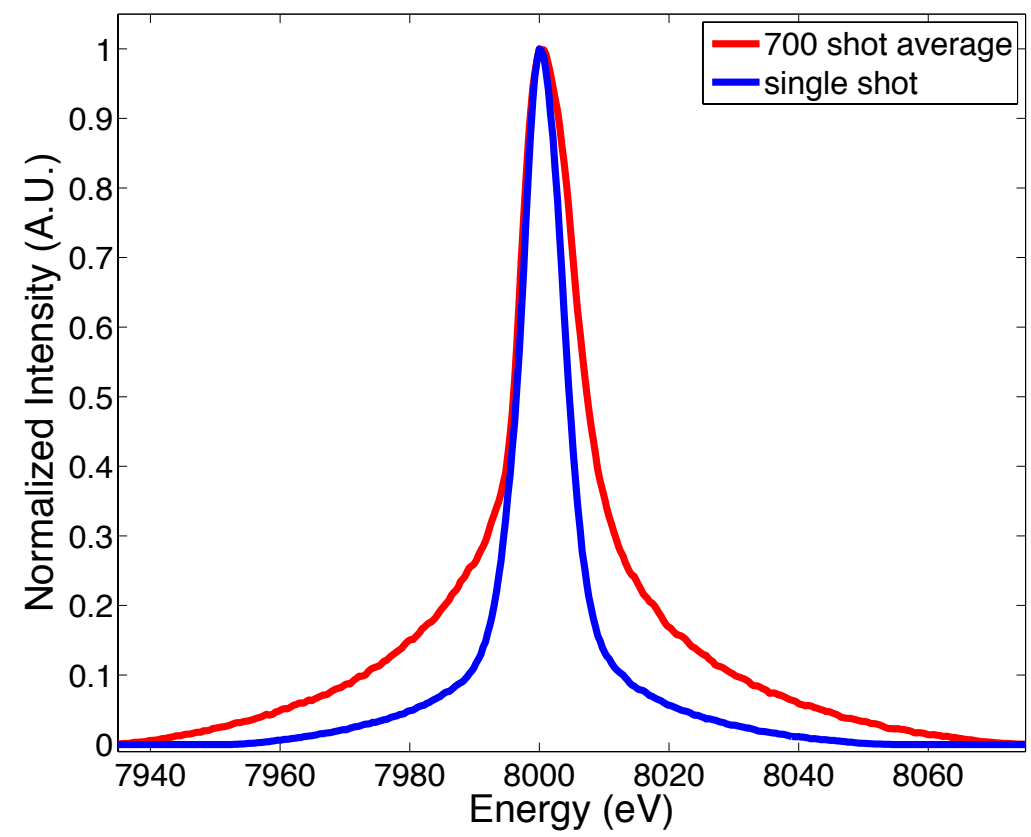

Figure 3. The HAPG instrument function is shown for the $40 \mathrm{~mm}$ thick crystal used for forward (plasmon) scattering experiments. The single shot profile is compared with the one obtained when averaging of 700 seeded shots with slightly fluctuating energy at a high-repetition rate. 
Figure 3 shows the instrument function derived from the ray tracing analysis. In separate experiments, excellent agreement with the measured shape of $\mathrm{K}-\alpha$ emission lines has been observed. Also shown is the instrument function applicable for experiments that average over 700 shots with a seeded x-ray beam. Here, we observe that statistical fluctuation in the x-ray laser energy result into an additional broadening of the effective profile. Even when taking these fluctuations into account the experiment is suited for resolving the plasmon frequency shift. Future improvements in x-ray instrumentation will be required for measurements of the spectral shape to determine damping of plasmon oscillations.

\section{Results and Discussions}

Figure 4 shows the experimental scattering spectra from solid-density aluminum. Results are shown from the forward scattering and backward scattering spectrometers with the $\mathrm{x}$-ray beam in seeded and SASE mode of operation. The seeded $\mathrm{x}$-ray beam provides a bandwidth of $1 \mathrm{eV}$ and combined with $8 \mathrm{eV}$ spectrometer resolution resolves the plasmon that is down-shifted from the elastic $8 \mathrm{keV}$ scattering feature by $19 \mathrm{eV}$. The figure also shows the results of the backward scattering spectrometer which observes elastic scattering at $8 \mathrm{keV}$ reflecting the instrument function of the spectrometer convolved with a Gaussian profile that accounts for the statistical fluctuations of the x-ray energy of a seeded beam over 700 shots.
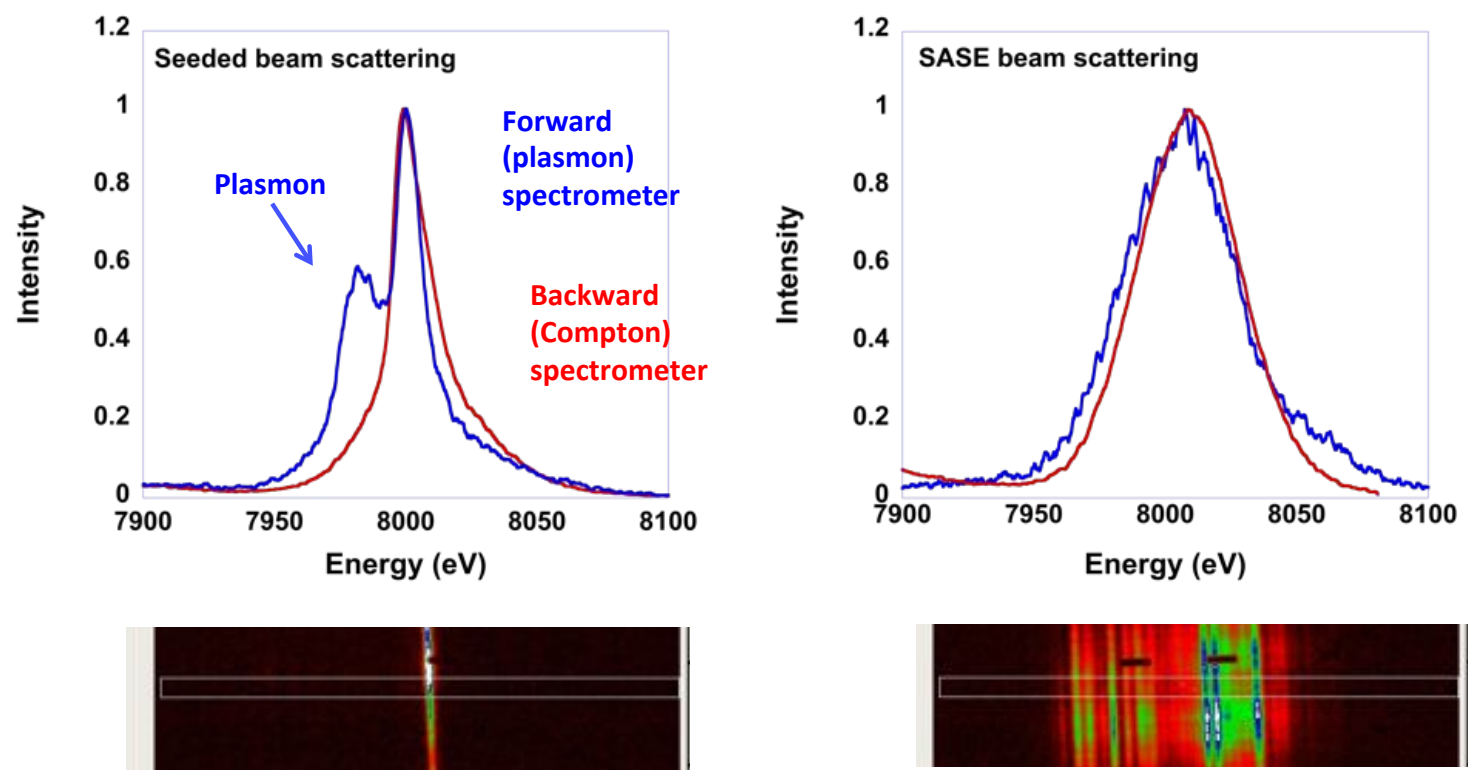

Seeded

Bent crystal spectrometer in MEC

SASE

Figure 4. X-ray Thomson scattering data from solid density aluminum targets are shown from forward (plasmon) scattering and backscattering spectrometers for seeded and SASE x-ray beam operation. These data are accumulated over 700 shots at $120 \mathrm{~Hz}$. Also shown are examples of the spectrum of a single x-ray laser shot indicating $1 \mathrm{eV}$ bandwidth for seeded beam and $50 \mathrm{eV}$ for SASE operations.

The backward spectrometer also measured a Compton scattering feature downshifted in energy by $250 \mathrm{eV}$ (not shown). Here, we note that the plasmon resonance is only observed in forward scattering providing strong evidence of a collective phenomenon as predicted for these 
conditions; bound-free scattering features are predicted to be negligible in this energy range and no feature has been observed with the Compton spectrometer. For these measurements, the crystal in the forward scattering spectrometer is $40 \mu \mathrm{m}$ thick while in backscattering we employed a $100 \mu \mathrm{m}$ thick crystal giving rise to slightly different instrument functions and consequently slight differences in the spectral shape of the elastic scattering feature.

Because the x-ray seed power is generated from the random SASE process that fluctuates in energy and amplitude, the output $x$-ray energy fluctuates nearly by $100 \%$. About $1 / 3$ of the shots provide sufficient photons with adequate spectrum for single shot $\mathrm{x}$-ray scattering experiments. When summing over 700 shots the spectrum shows a broad foot, but the bandwidth is mostly preserved greatly improving the resolution when compared to the SASE spectrum. In forward direction, the x-ray scattering spectra with SASE operation show a slight broadening of red wing of the scattering spectrum but the SASE scattering spectrum is not suitable for inferring the dense plasma conditions.

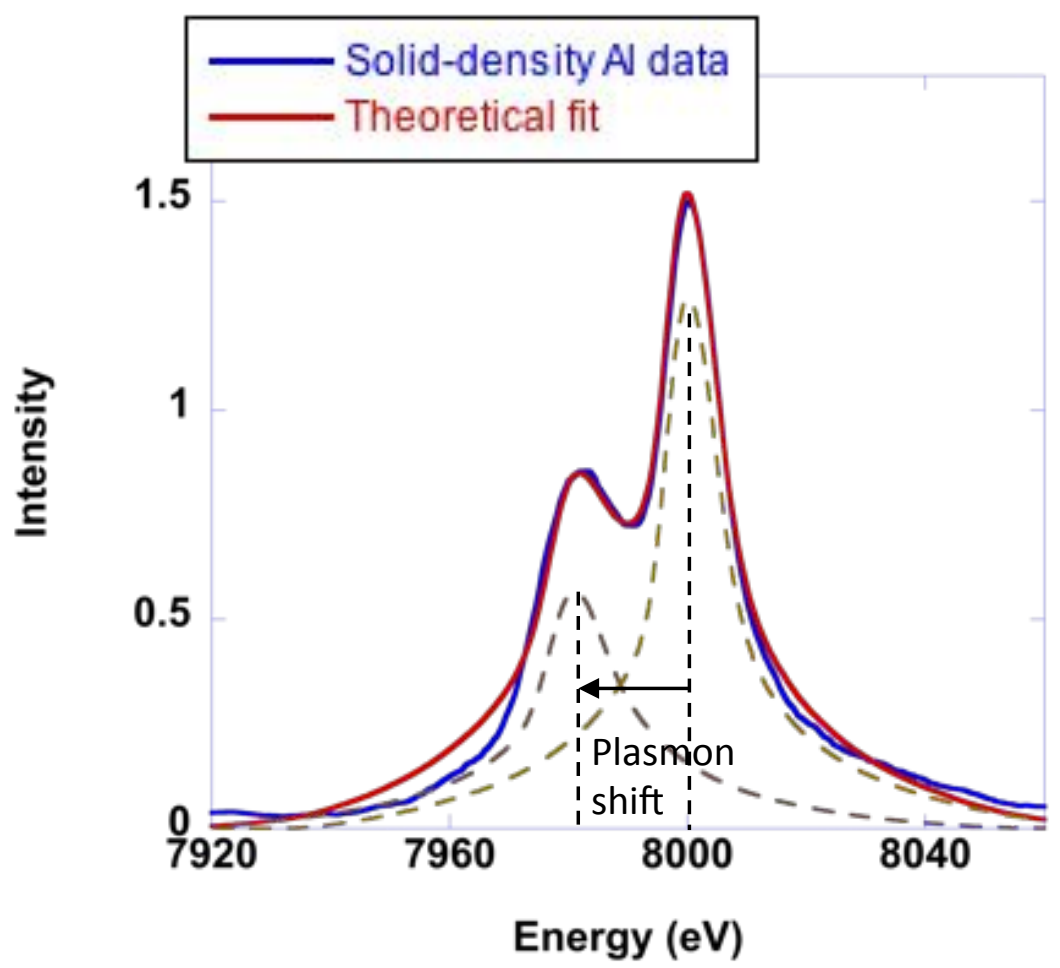

Figure 5. The theoretical fit to the experimental forward X-ray Thomson scattering data is shown for the electron density of $n_{e}=1.8 \times 10^{23} \mathrm{~cm}^{-3}$. The theoretical fit uses an electron temperature of $2 \mathrm{eV}$ reflecting the direct heating of electrons by the x-ray laser interactions. The ions are assumed cold with temperatures of order $0.1 \mathrm{eV}$. The red dashed curve shows the elastic scattering component and the blue dashed curve represents the plasmon spectrum.

Figure 5 shows the theoretical fit of the dynamic structure factor, equation (3) to the experimental forward scattering spectrum from Fig. 4. The shift of the plasmon peak yields the electron density of $\mathrm{n}_{\mathrm{e}}=1.8 \times 10^{23} \mathrm{~cm}^{-3} \pm 5 \%$. The electron temperature of the fit is $2 \mathrm{eV}$ accounting for the isochoric heating of the aluminum by the x-ray laser pulse [30]. The ions are cold with temperatures of order $0.1 \mathrm{eV}$ consistent with the observation of Debye-Scherrer rings 
from solid aluminum. Here, the plasmon frequency is very sensitive to the electron density, but the temperatures are too small to affect the plasmon. The accuracy of the electron density measurement is very high due to the sensitivity of the plasmon resonance to the plasma frequency. With $n_{e}=Z / A 6.02 \times 10^{23} \rho$ (the electron density $n_{e}$ in $\mathrm{cm}^{-3}$ and the mass density $\rho$ in $\mathrm{g} \mathrm{cm}^{-3}$ ), assuming ionization state of $Z=3$ and using the mass $A=26.98 \mathrm{u}$, the measured electron density provides $\rho=2.7 \mathrm{~g} \mathrm{~cm}^{-3}$ as expected for solid aluminum. In these warm dense matter conditions, knowledge of the ion-ion structure factor is limited and inferring the temperature from, e.g., the intensity ratio of the plasmon feature to the elastic scattering feature will await future experimental tests of the theoretical description of the interaction potentials.

\section{Conclusions}

We have demonstrated plasmon measurements in dense matter using the seeded LCLS xray beam. The high bandwidth and high photon numbers combined with high-resolution HAPG crystals allows resolving the plasmon shift of $19 \mathrm{eV}$ resulting in an electron density $\mathrm{n}_{\mathrm{e}}=1.8 \mathrm{x}$ $10^{23} \mathrm{~cm}^{-3} \pm 5 \%$. This density is consistent with $\mathrm{Z}=3$ in solid aluminum. For future experiments this capability can be applied in single shots to determine, e.g., the conditions of warm dense matter that is compressed by laser-driven shock waves. Moreover, precision measurements of the plasmon dispersion and amplitude will now be possible. Future developments will include high-resolution spectrometers with sufficient efficiency for measuring the plasmon broadening through Landau and collisional damping processes.

\section{Acknowledgments}

This work was performed at the Matter at Extreme Conditions (MEC) instrument of LCLS, supported by the DOE Office of Science, Fusion Energy Science under contract No. SF00515. This work was partially supported by DOE Office of Basic Energy Sciences, Materials Sciences and Engineering Division, under Contract DE-AC02-76SF00515. UZ is grateful to the VolkswagenStiftung for his Peter-Paul-Ewald Fellowship.

\section{References}

[1] P. Emma et al., First lasing and operation of an ångstrom-wavelength free-electron laser, Nature Photonics 4, (2010) 641 - 647.

[2] H.-J. Lee et al., to be published

[3] S. H. Glenzer and R. Redmer, X-ray Thomson scattering in high energy density plasmas, Rev. Mod. Phys. 81 (2009) 1625.

[4] F. Coppari et al., Experimental evidence for a phase transition in magnesium oxide at exoplanet pressures, Nature Geoscience (2013) doi:10.1038/ngeo1948.

[5] A. M. Lindenberg et al., Time-Resolved X-Ray Diffraction from Coherent Phonons during a LaserInduced Phase Transition, Phys. Rev. Lett. 84 (2000) 111.

[6] C. Fortmann, C. Niemann, and S. H. Glenzer, Theory of x-ray scattering in high-pressure electrides, Phys. Rev. B 86 (2012) 174116. 
[7] S. H. Glenzer. et al., Observations of Plasmons in Warm Dense Matter, Phys. Rev. Lett. 98 (2007) 065002 .

[8] A. L. Kritcher et al., Ultrafast X-ray Thomson scattering of shock-compressed matter, Science $\mathbf{3 2 2}$ (2008) 69.

[9] P. Neumayer et al., Plasmons in strongly coupled shock-compressed matter, Phys. Rev. Lett. 105 (2010) 075003.

[10] G. Gregori and D. O. Gericke, Low frequency structural dynamics of warm dense matter, Physics of Plasmas 16, (2009) 056306.

[11] T. Döppner et al., Temperature measurement through detailed balance in x-ray Thomson scattering, High Energy Density Physics 5 (2009) 182-186.

[12] B. M. Hegelich et al., Laser acceleration of quasi-monoenergetic MeV ion beams, Nature (2006) 441.

[13] Y. Sentoku et al., Dynamic Control over Mega-Ampere Electron Currents in Metals Using Ionization-Driven Phys. Rev. Lett. 107 (2011) 135005.

[14] J. D. Lindl et al., The physics basis for ignition using indirect-drive targets on the National Ignition Facility, Physics of Plasmas 11, (2004) 339.

[15] S. H. Glenzer et al., The physics basis for ignition using indirect-drive targets on the National Ignition Facility, Physics of Plasmas 19, (2012) 056318.

[16] R. Blandford and D. Eichler, Particle acceleration at astrophysical shocks: A theory of cosmic ray origin. Phys. Rep. 154, (1987) 1.

[17] N. L. Kugland, et al., Self-organized electromagnetic field structures in laser-produced counterstreaming plasmas, Nature Physics, (2012) DOI: 10.1038/NPHYS2434.

[18] H.-J. Lee et al., X-Ray Thomson-Scattering Measurements of Density and Temperature in ShockCompressed Beryllium, Phys. Rev. Lett. 102 (2009) 115001.

[19] C. Fortmann et al., Measurement of the Adiabatic Index in Be Compressed by Counterpropagating Shocks, Phys. Rev. Lett. 108 (2012) 175006.

[20] J. Chihara, "Difference in x--ray scattering between metallic and non--metallic liquids due to conduction electrons", J. Phys. F: Met. Phys. 17, (1987) 295.

[21] G. Gregori et al., Theoretical model of x-ray scattering as a dense matter probe, Phys. Rev. E. 67, (2003) 026412.

[22] G. Gregori et al., Generalized x-ray scattering cross section from non-equilibrium solids and plasmas, Phys. Rev. E. 74, (2006) 026402.

[23] R. Zimmerman, Many-Particle Theory of Highly Excited Semiconductors (Teubner, Leipzig, 1987).

[24] R. Thiele et al., Plasmon resonance in warm dense matter Phys. Rev. E. 78, (2008) 026411.

[25] J. Amann et al., Demonstration of self-seeding in a hard-X-ray free-electron laser, Nature Photonics (2012) DOI: 10.1038/NPHOTON.2012.180 
[26] A. Pak et al., X-ray line measurements with high efficiency Bragg crystals, Rev. Sci. Instrum. 75 (2004) 3747.

[27] U Zastrau, A. Woldegeorgis, et al., Characterization of strongly bent HAPG crystals for von-Hamos x-ray spectrographs, J. Instrumentation (2013) 8 P10006 DOI:10.1088/1748-0221/8/10/P10006

[28] H. Legall et al., New generation of x-ray optics based on pyrolytic graphite, Proceedings of FEL 2006, BESSY, Berlin, Germany.

[29] U. Zastrau et al., Focal aberrations of large-aperture HOPG von-Hamos x-ray spectrometers, J. Instrumentation (2012) 7 P09015 DOI: 0.1088/1748-0221/7/09/P09015.

[30] S. Hau-Riege et al., Ultrafast Transitions from Solid to Liquid and Plasma States of Graphite Induced by X-Ray Free-Electron Laser Pulses, Phys. Rev. Lett. 108 (2012) 217402. 